\title{
Irradiation of silver and agar/silver nanoparticles with argon, oxygen glow discharge plasma, and mercury lamp
}

\author{
Mahmoud M Ahmad ${ }^{1 *}$, Essam A Abdel-Wahab ${ }^{1}$, A A El-Maaref ${ }^{1}$, Mohammed Rawway $^{2}$ and Essam R Shaaban ${ }^{1}$
}

\begin{abstract}
The irradiation effect of argon, oxygen glow discharge plasma, and mercury lamp on silver and agar/silver nanoparticle samples is studied. The irradiation time dependence of the synthesized silver and agar/silver nanoparticle absorption spectra and their antibacterial effect are studied and compared. In the agar/silver nanoparticle sample, as the irradiation time of argon glow discharge plasma or mercury lamp increases, the peak intensity and the full width at half maximum, FWHM, of the surface plasmon resonance absorption band is increased, however a decrease of the peak intensity with oxygen glow plasma has been observed. In the silver nanoparticle sample, as the irradiation time of argon, oxygen glow discharge plasma or mercury lamp increases, the peak intensity of the surface plasmon resonance absorption band is increased, however, there is no significant change in the FWHM of the surface plasmon resonance absorption band. The SEM results for both samples showed nanoparticle formation with mean size about $50 \mathrm{~nm}$ and $40 \mathrm{~nm}$ respectively. Throughout the irradiation time with the argon, oxygen glow discharge plasma or mercury lamp, the antibacterial activity of several kinds of Gram-positive and Gram-negative bacteria has been examined.
\end{abstract}

Keywords: Silver nanoparticles; Glow discharge plasma; UV light irradiation; Antibacterial activity

\section{Introduction}

Due to their unique properties, metal nanoparticles are attracted a great interest of experimental and theoretical investigations (Amoruso et al. 2005; Link and El-Sayed 2000; Richardson et al. 2009; Shalaev 2002). Controlling of metalic nanoparticles geometry has found special interset since this allows tuning optical properties that are not present in bulk materials (Ahmadi et al. 1996). The tunability of the plasmon position and its charachterstics such as Full width at half maximum (FWHM), and peak intensity makes the nanoparticles attractive for several applications (Cobley et al. 2009; McFarland and Van Duyne 2003). The mainpulation of nanoparticles can be achieved with laser, UV-light as well as with plasma sources (Hou et al. 2013; Mafune et al. 2000; Zhen et al. 2013). In principle, irradiation with a given light source excites and heats nanoparticles of certain sizes or/and shapes and leads to diffusion and evaporation of surface

\footnotetext{
*Correspondence: m_ahmed@azhar.edu.eg

'Physics Department, Faculty of science, Al-Azhar University-Assuit branch, Assuit, Egypt

Full list of author information is available at the end of the article
}

atoms. Thus, tuning the plasmon position and its charachterstics of the nanoparticles can be accomplished. In the present experiment, silver and agar/silver nanoparticles were prepared using chemical reduction method (Pillai and Kamat 2004). The prepared samples have been irradiated with argon, oxygen glow discharge plasma sources, and mercury lamp at different time periods. Spectrophotometric measurements were carried out to follow the irradiation process and to characterize the optical properties of the resultant silver and agar/silver nanoparticles. Finally, the resultant nanoparticles samples have been examined for antibacterial activity against various types of Gram-positive and Gram-negative bacteria, which are necessary in order to fully evaluate its possible use as a new bactericidal material.

\section{Methods}

Synthesis

The silver nanoparticle samples have been prepared by using chemical reduction method. All solutions of reacting materials have been prepared in distilled water. Silver nitrate $\mathrm{AgNO}_{3}$ and trisodium citrate $\mathrm{C}_{2} \mathrm{H}_{5} \mathrm{O}_{7} \mathrm{Na}_{3}$ of 
analytical grade purity were used as starting materials without further purification. In the present procedure $150 \mathrm{~mL}$ of $1 \mathrm{mM} \mathrm{AgNO} 3$ was heated to boiling and $15 \mathrm{~mL}$ of $1 \%$ trisodium citrate was added drop by drop to the solution until its color change to pale yellow. Then it was removed from the heating plate and stirred until cooled to room temperature and it kept in dark place. The agar/Silver nanoparticles sample has been prepared by adding $0.1 \mathrm{gm}$ of agar powder to $10 \mathrm{~mL}$ of silver nanoparticles solution and stirred for two minutes at room temperature.

For SEM analysis, samples are prepared by depositing a drop of colloidal solution on a carbon coated copper SEM holder and drying at room temperature. Absorption spectra were recorded at room temperature using Jasco-670 double beam spectrometer.

\section{Discharge plasma setup and Irradiation procedures}

In order to setup argon, oxygen glow discharge plasma sources, two copper circular plane electrodes are used. The two electrodes are centered in the reaction chamber axes. The gas has injected into the reaction chamber through the side flange. The reaction chamber was evacuated up to $10^{-3} \mathrm{mmHg}$ before the gas inlet. The gas pressure has controlled using vacuum system and gauges to $0.11 \mathrm{mmHg}$ and kept constant during the measurement procedure. The discharge voltages of the argon and oxygen plasma were 248 and 358 volt respectively. More details of the plasma source setup can be found in (Shaaban et al. 2013). Figure 1 shows a schematic diagram of the discharge plasma setup. The strong emission spectral lines of argon, oxygen glow discharge plasma sources and mercury light source are listed in Table 1 (Reader et al. 1996; Bacławski and Musielok 2008).

Six samples of the same volume $3 \mathrm{~mL}$ of silver and agar/ silver nanoparticles have been irradiated with argon, oxygen glow discharge plasma sources, and mercury lamp at different time periods. Spectrophotometric measurements were carried out to follow the irradiation process and to characterize the optical properties of the resultant silver and agar/silver nanoparticles.

\section{Antibacterial procedures}

The disc diffusion assay method was used to study the antibacterial activity of the synthesized nanoparticle samples (NCCLS 1993). All the glassware, media and reagents used were sterilized in an autoclave at $121^{\circ} \mathrm{C}$ for $20 \mathrm{~min}$.

The antibacterial activity of nanoparticle samples was evaluated against some of Gram positive (Bacillus cereus, Bacillus subtilis, Micrococcus roseus, Staphylococcus aureus and Streptococcus sp.) and Gram negative bacteria (E.coli, Klebsiella pneumoniae, Proteus vulgaris, Pseudomonas aeruginosa and Serratia marcescens). Bacterial suspension was prepared by growing a single colony overnight in nutrient broth and by adjusting the turbidity to 0.5 McFarland standards (Kora et al. 2009).

Media plates were inoculated with this bacterial suspension. The sterile filter-paper disks (Whatman filter paper no.1) of $6 \mathrm{~mm}$ were impregnated with nanoparticle samples solution and placed on the surface of the media inoculated with bacterial species. These plates were incubated at $37^{\circ} \mathrm{C}$ for $24 \mathrm{~h}$ and the zone of inhibition (ZOI) was measured.

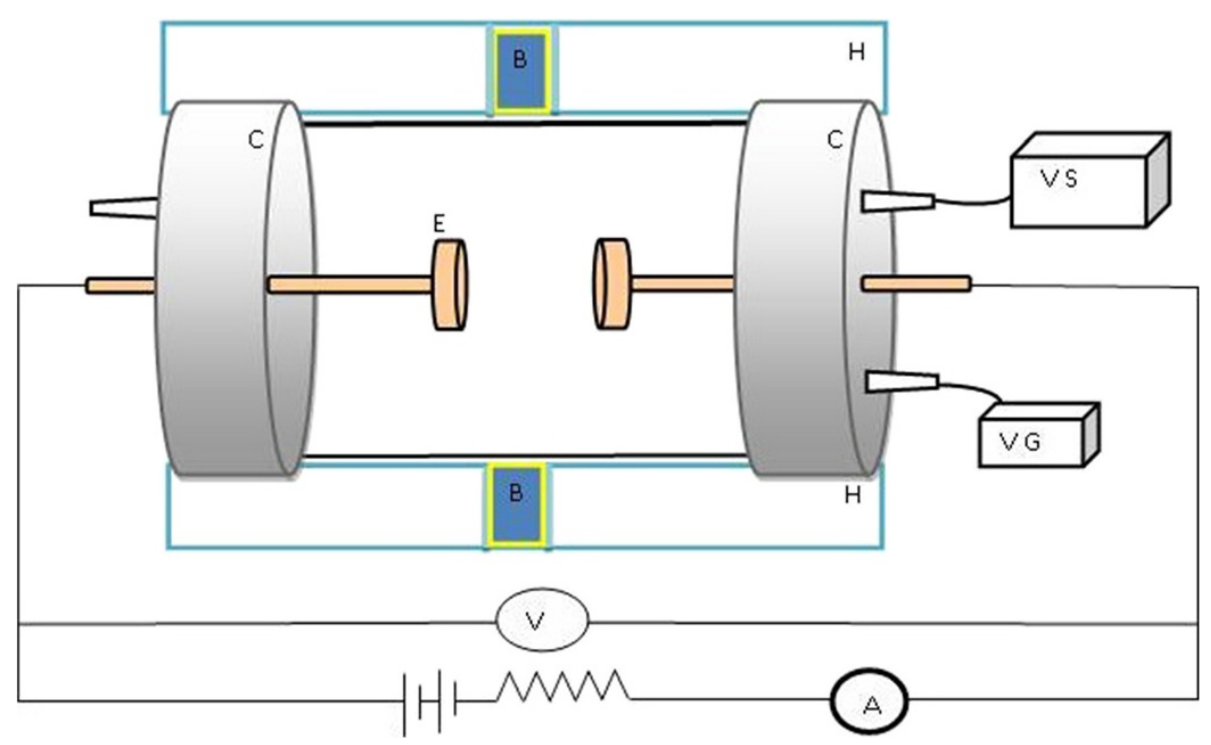

Figure 1 Schematic diagram of an electric discharge cell. Where $E$ is two copper circular plane electrodes $(0.2 \mathrm{~cm}$ thickness and $5 \mathrm{~cm}$ diameter), $C$ is the reaction chamber (cylindrical Pyrex glass of $7 \mathrm{~cm}$ diameter and $15 \mathrm{~cm}$ length with $2 \mathrm{~cm}$ gap spacing, VS is the vacuum system, VG is the vacuum gauge, $\mathrm{H}$ is the sample holder, and $\mathrm{B}$ is cuvette place holder. 
Table 1 The strongest emission lines in $\mathrm{nm}$ of argon, oxygen glow discharge plasma sources and mercury light source (Bacławski and Musielok 2008; Reader et al. 1996)

\begin{tabular}{ccc}
\hline $\begin{array}{c}\text { Oxygen lines } \\
(\mathbf{n m}) \text { O I }\end{array}$ & $\begin{array}{c}\text { Mercury lines } \\
(\mathbf{n m}) \mathbf{H g ~ I}\end{array}$ & $\begin{array}{c}\text { Argon lines } \\
(\mathbf{n m}) \text { Ar I }\end{array}$ \\
\hline 777.337 & 253.652 & 696.543 \\
645.499 & 296.728 & 706.722 \\
725.436 & 302.150 & 714.704 \\
615.727 & 313.155 & 727.294 \\
715.670 & 334.148 & 738.398 \\
926.387 & 365.015 & 750.387 \\
948.289 & 404.656 & 763.511 \\
949.794 & 407.783 & 772.376 \\
950.560 & 435.833 & 794.818 \\
949.271 & 546.074 & 826.452 \\
948.743 & 576.960 & 842.465 \\
& 579.066 & 852.144 \\
& & 866.794 \\
& & 912.297 \\
& & 922.450 \\
\hline
\end{tabular}

\section{Results and discussions}

SEM analysis has been performed in order to observe morphology of the synthesized samples. Figures 2 and 3 show SEM images and the corresponding size distributions of silver and agar/silver nanoparticle samples respectively. From SEM images the silver and agar/silver nanoparticles are spherically shaped with mean size about $50 \mathrm{~nm}$ and $40 \mathrm{~nm}$ respectively. The effect of agar powder on the synthesized silver nanoparticles states as controller of nucleation as well as stabilizer.

Measurements of UV-vis spectrophotometer show the expected surface plasmon resonance SPR peak of silver nanoparticles. In the UV-vis spectra a single strong peak with a maximum around $424 \mathrm{~nm}$ is observed of silver nanoparticle samples, which corresponds to the typical SPR of conducting electrons of the surface of silver nanoparticles. In agar/silver nanoparticle samples a single strong peak with a maximum around $428 \mathrm{~nm}$ has been detected. This shift of peak position of the SPR band between the two samples is due to the sensitivity of SPR to the shape, size, and interaction of the particle with the medium and local refractive index.

It is observed that, there are no peaks located around 335 and $560 \mathrm{~nm}$, which indicate to the complete absence of nanoparticle aggregation (Mohan et al. 2007; Kora et al. 2009).

Figures 4, 5, and 6 show the absorption spectrum of silver nanoparticle samples irradiated with argon, oxygen, and mercury lamp at different time intervals respectively. It is shown that, in the case of silver nanoparticle sample as the irradiation time of argon, oxygen glow discharge plasma or mercury lamp increases, the peak intensity of the SPR absorption band is increased. However, there is no significant change of the FWHM at the SPR absorption band.

Figures 7, 8, and 9 show the absorption spectrum of agar/silver nanoparticle samples irradiated with argon, oxygen, and mercury lamp at different time intervals respectively. It is shown that for agar/silver nanoparticle sample as the irradiation time of argon glow discharge plasma or mercury lamp increases, the peak intensity and the FWHM of the SPR absorption band are increased. However a decrease of the peak intensity with oxygen glow discharge plasma is observed and the FWHM remains constant.

Variation of SPR peak position of silver and agar/silver nanoparticle samples after irradiation with argon, oxygen plasma and mercury lamp have been plotted in Figures 10, 11 , and 12 respectively. It is shown that, the SPR peak position remains constant in the case of silver nanoparticle

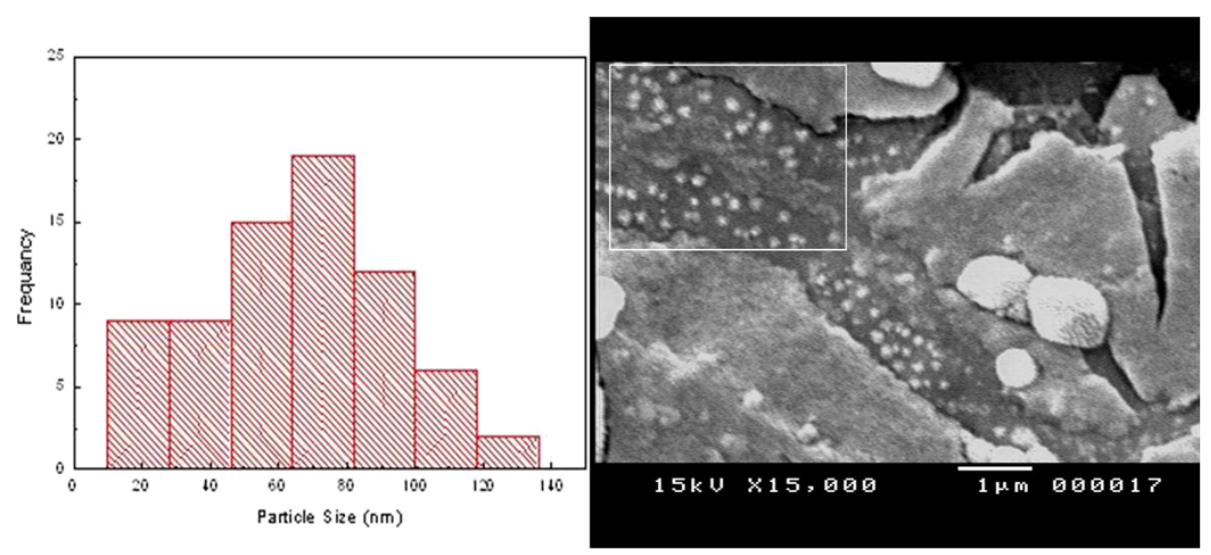

Figure 2 SEM image and particle size histogram of silver nanoparticles. 


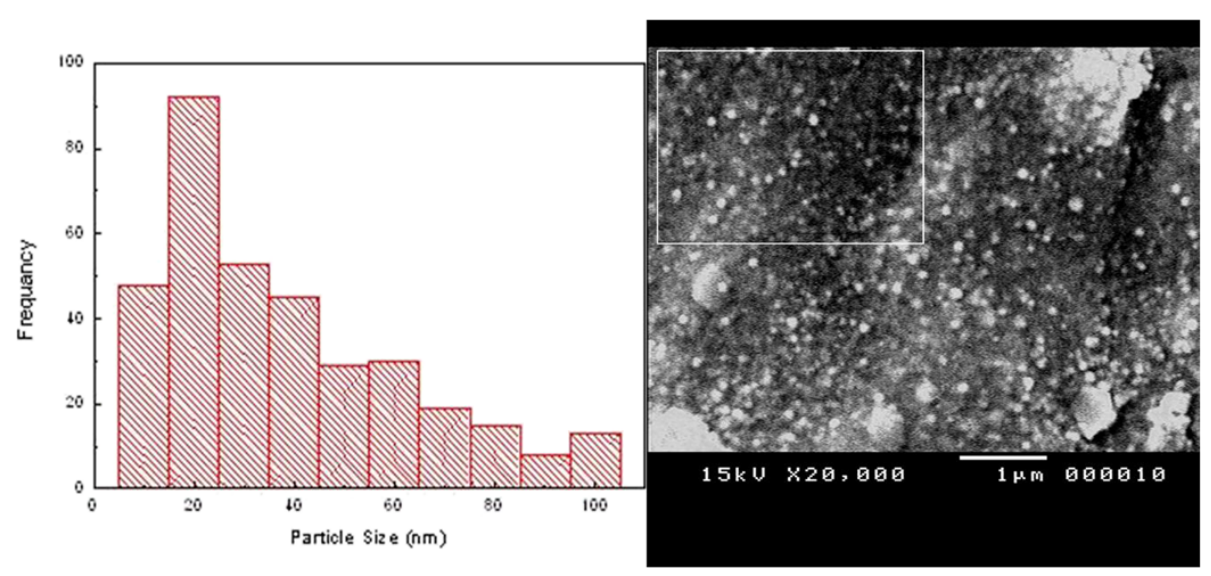

Figure 3 SEM image and particle size histogram of agar/silver nanoparticles.

sample irradiated with argon plasma, however a stepwise increment is observed in the case of agar/silver nanoparticle sample.

When both samples are irradiated with oxygen plasma, the SPR peak position remains constant.

It is observed that, the SPR peak position is increased for agar/silver nanoparticle sample after irradiation with mercury lamp. However there is no significant change of SPR peak position of silver nanoparticle sample.

Figures 13, 14, and 15 show the variation of the FWHM of silver and agar/silver nanoparticle samples

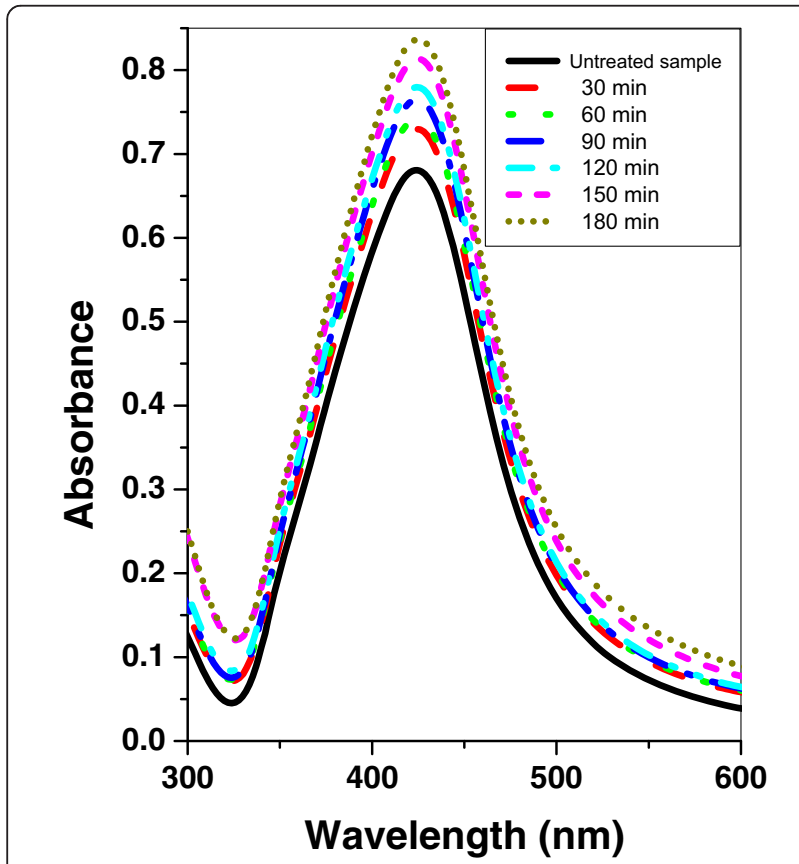

Figure $4 \mathrm{UV}$-vis absorbance spectra of silver nanoparticle sample before and after irradiation with argon plasma at different time periods. after irradiation with argon, oxygen plasma and mercury lamp respectively. It is observed that, there is no change of FWHM for silver nanoparticle sample irradiated with argon plasma however the FWHM is increased rapidly for agar/silver nanoparticle sample. In the case of oxygen plasma irradiation the FWHM remains constant for both samples.

After irradiation with mercury lamp the FWHM increases rapidly for silver nanoparticle sample; however there is a hysterics behavior of agar/silver nanoparticle sample.

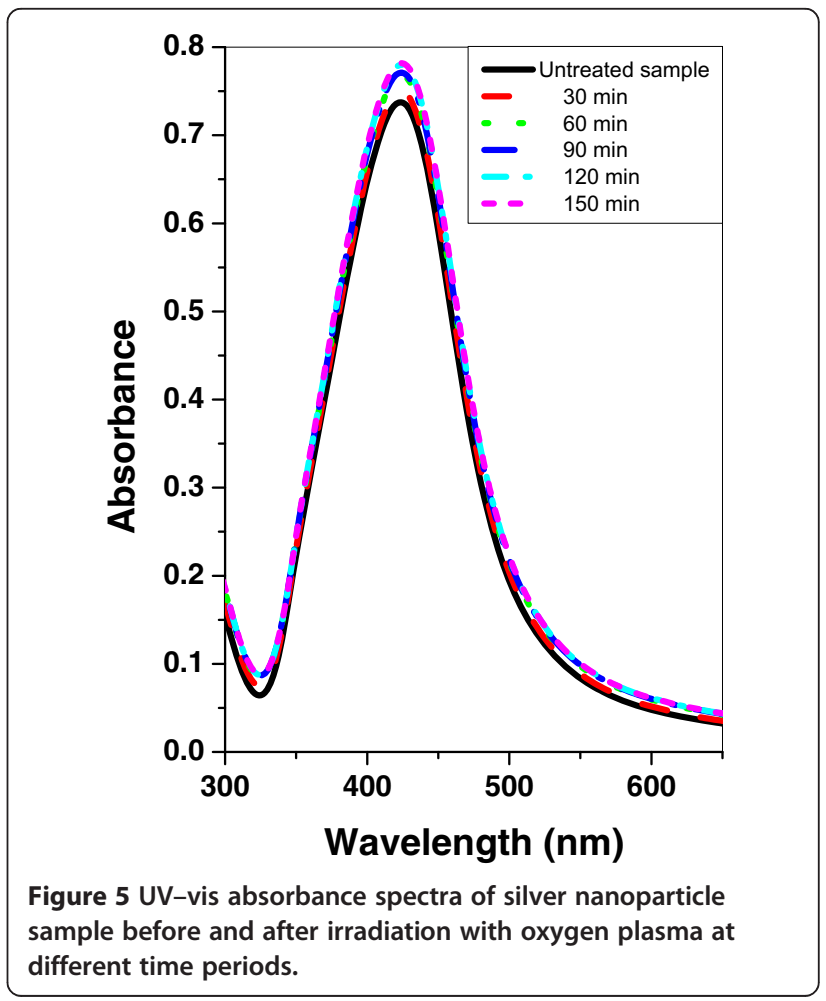




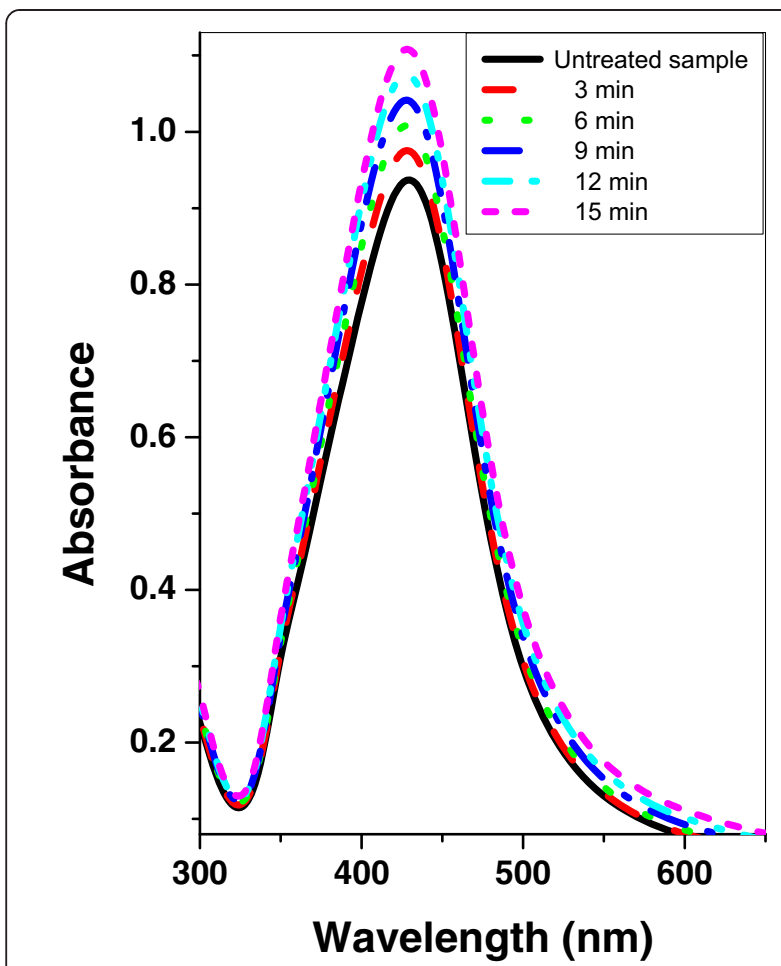

Figure 6 UV-vis absorbance spectra of silver nanoparticle sample before and after irradiation with mercury lamp at different time periods.

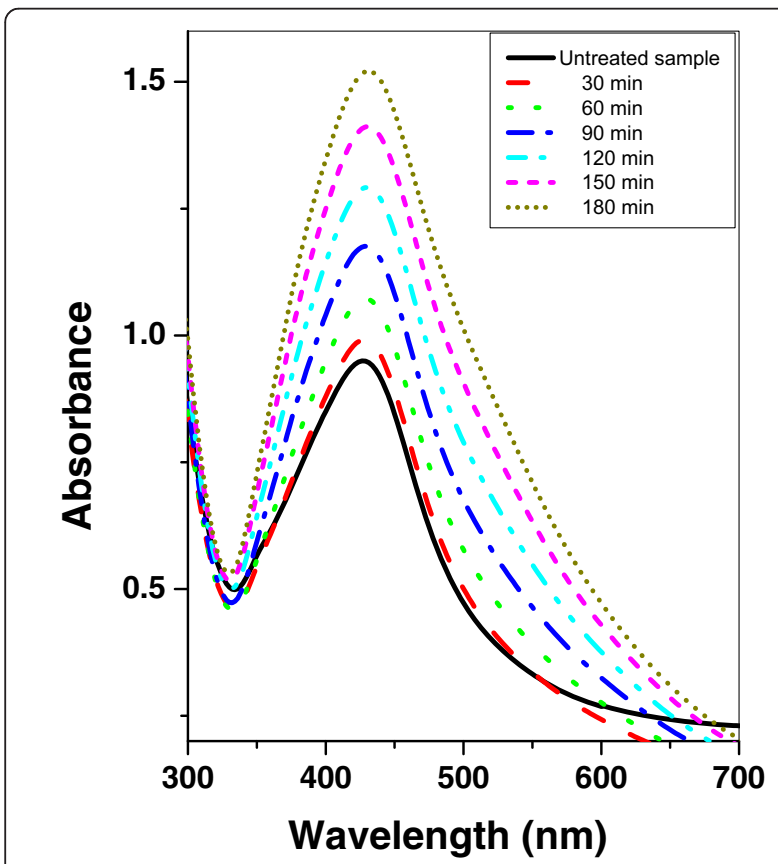

Figure $7 \mathrm{UV}$-vis absorbance spectra of agar/silver sample before and after irradiation with argon plasma at different time periods.

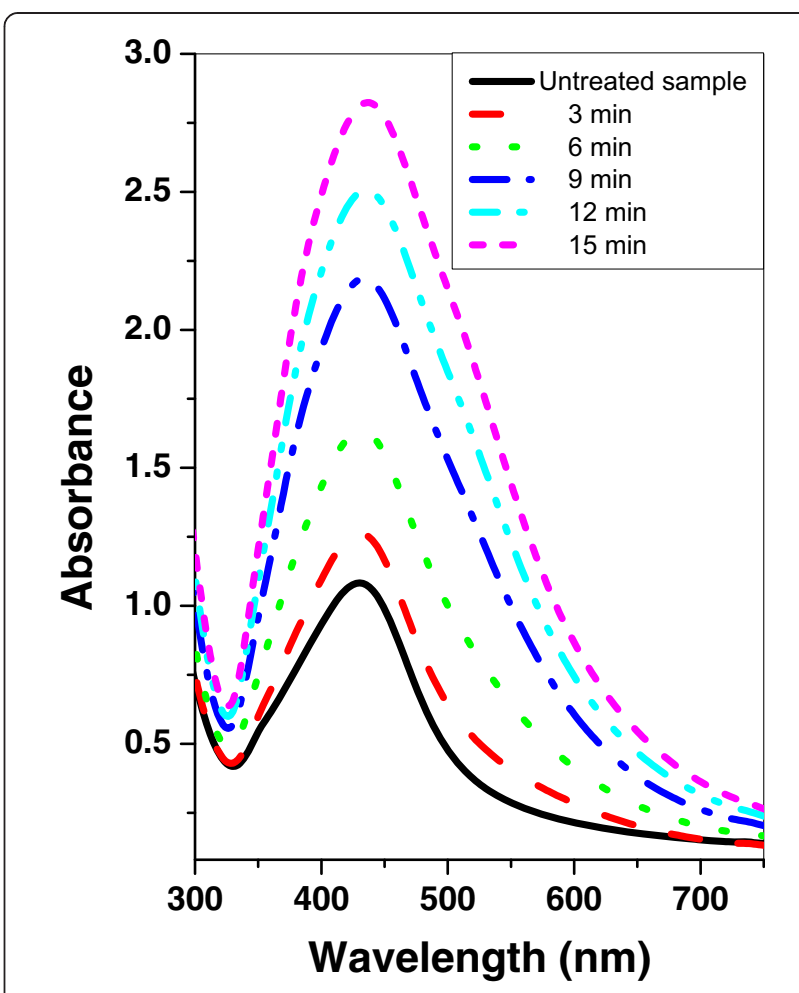

Figure 8 UV-vis absorbance spectra of agar/silver nanoparticle sample before and after irradiation with mercury lamp at different time periods.

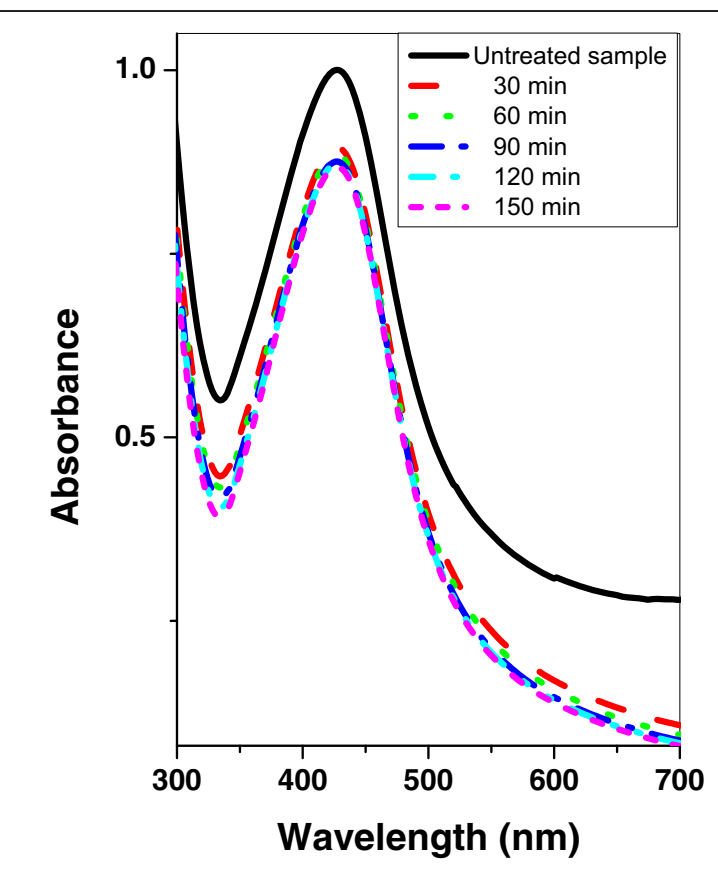

Figure 9 UV-vis absorbance spectra of agar/silver nanoparticle sample before and after irradiation with oxygen plasma at different time periods. 


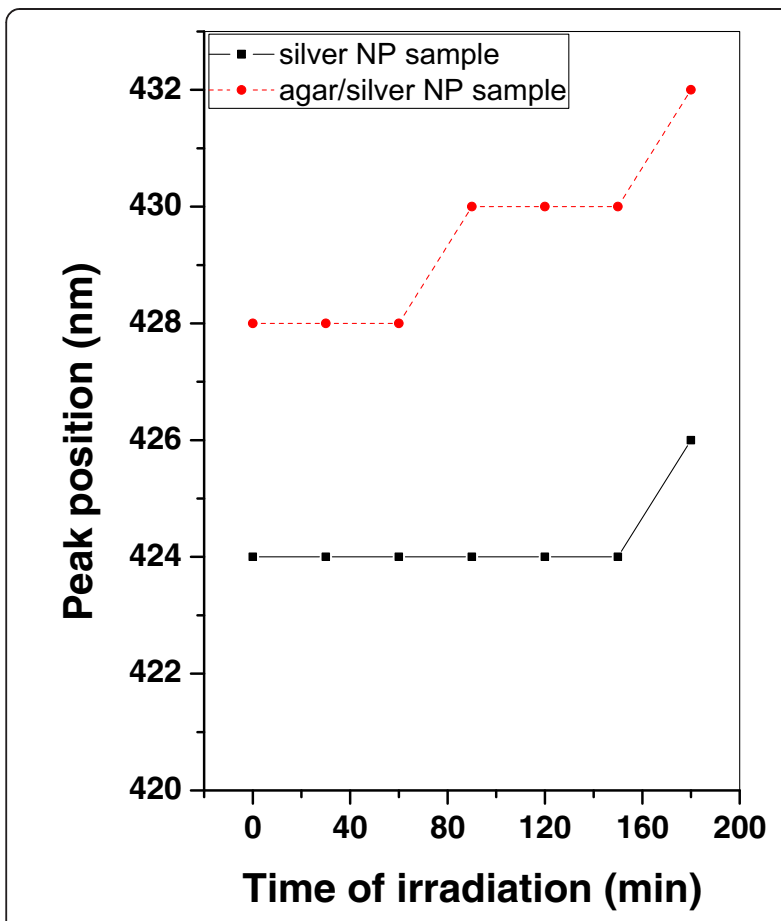

Figure 10 Variation of SPR peak position of silver and agar/silver samples after irradiation with argon plasma.

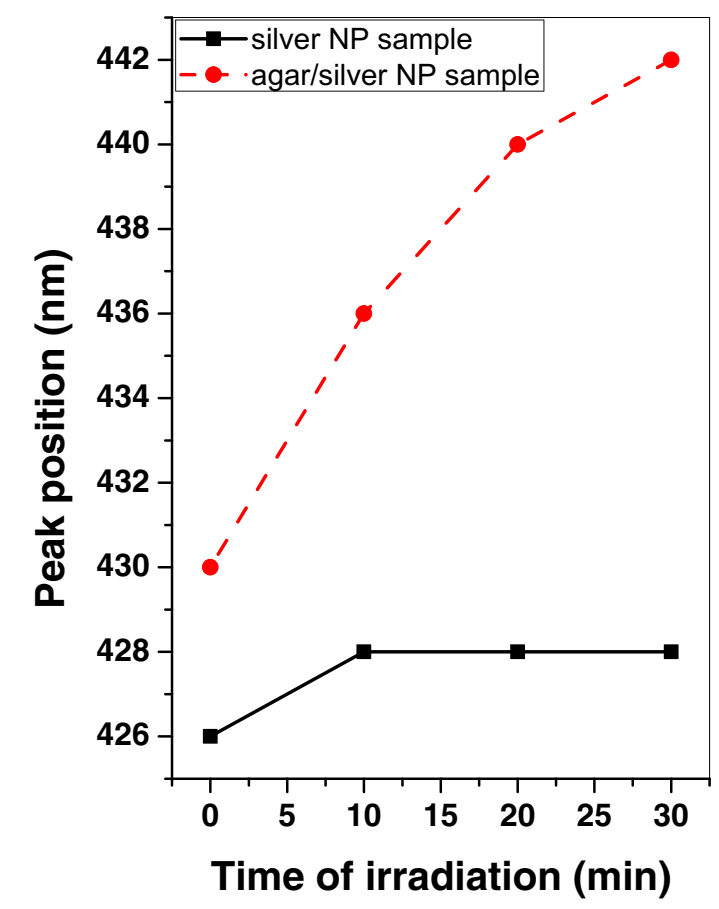

Figure 12 Variation of SPR peak position of silver and agar/silver samples after irradiation with mercury lamp.

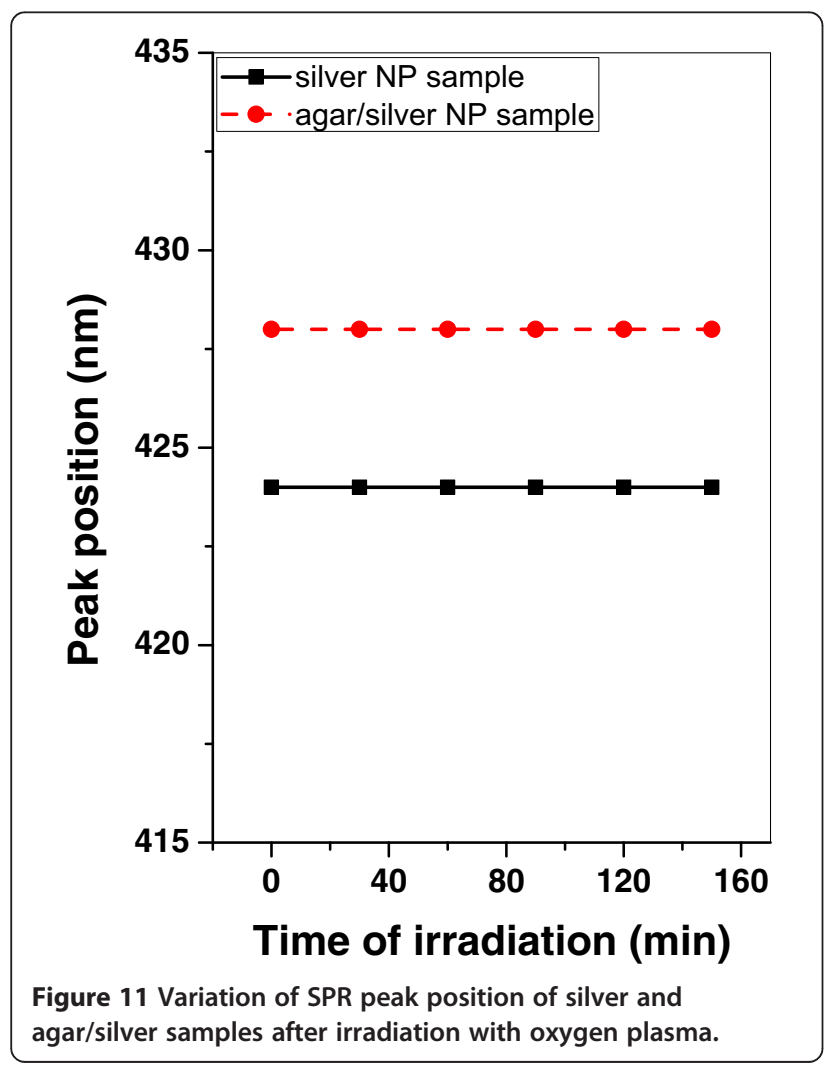

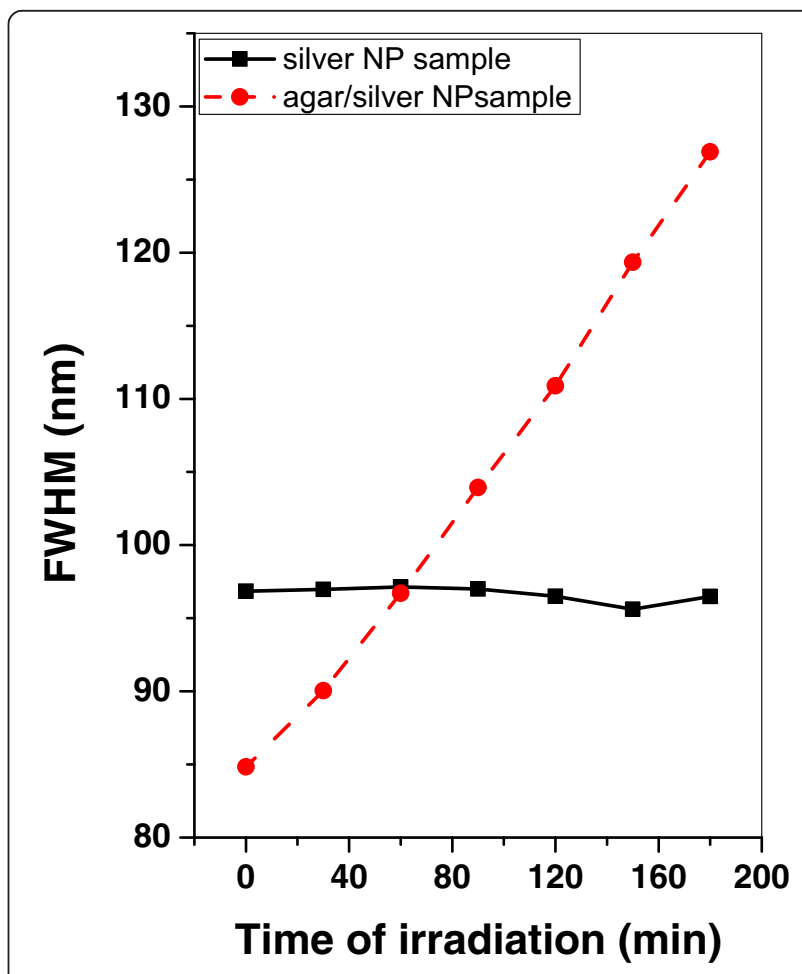

Figure 13 Variation of FWHM of silver and agar/silver samples after irradiation with argon plasma. 


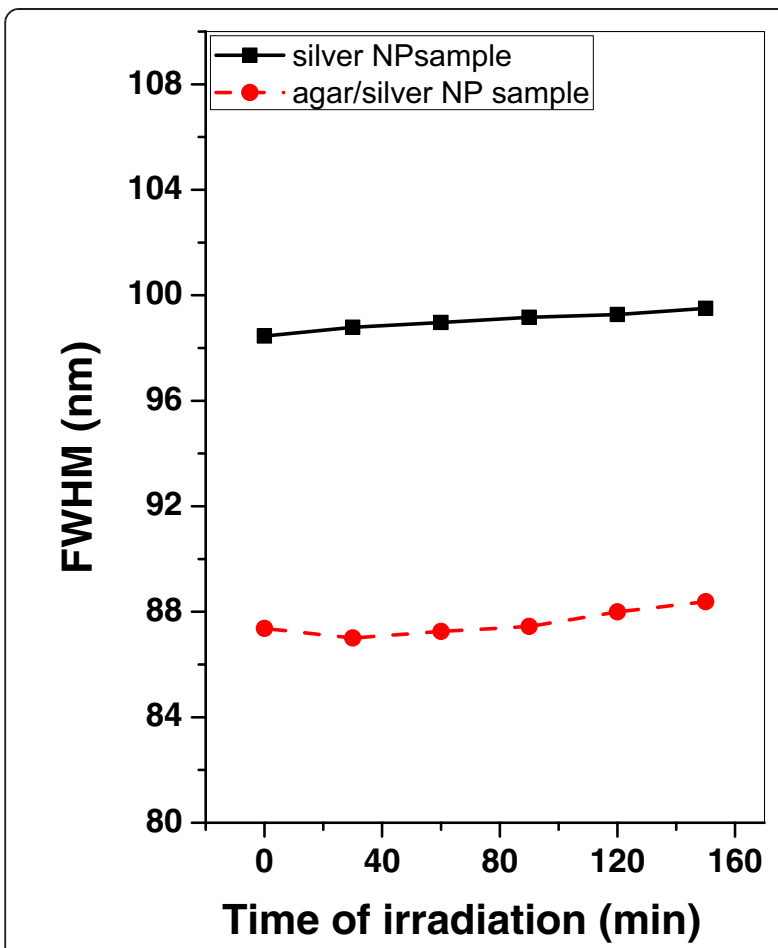

Figure 14 Variation of FWHM of silver and agar/silver samples after irradiation with oxygen plasma.

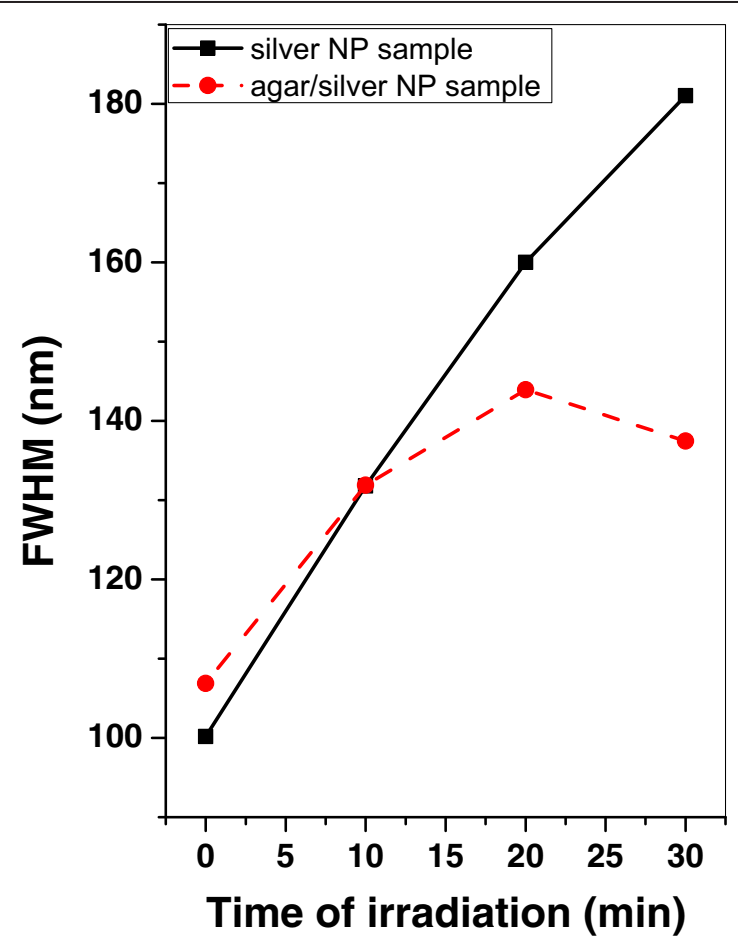

Figure 15 Variation of FWHM of silver and agar/silver samples after irradiation with mercury lamp.
The above results are discussed in terms of a mechanism in which, agar consists of a mixture of agarose and agaropectin. It is composed of alternating 1,3linked d-galactose and 1,4-linked 3,6 anhydro-l galactose units (Labropoulos et al. 2002). It has the ability to form reversible gels simply by cooling a hot aqueous solution.

It is well known that, polysaccharide contains of hydroxyl, acetyl, carbonyl and carboxylic functional groups. This disaccharide can be substituted by sulfate esters and methoxyl, and may also carry pyruvic acid residues (Duckworth and Yaphe 1971). The type, amount, and location of these substitutes strongly affect the physical properties of the gel and therefore, its functionality (Freile-Pelegrin and Murano 2005).

Based on these facts, it can be inferred that both hydroxyl and carbonyl groups of agar are involved in the synthesis of agar/silver nanoparticle sample and effectively help in capping the surface of nanoparticles. The variations in the shape and peak position of the hydroxyl and carboxylate groups using FTIR have been reported (Guerrero et al. 2014). Also silver nanoparticles can synthesized using another polysaccharide i.e. gum Acacia (Mohan et al. 2007), gum kondagogu (Kora et al. 2010) and gum Arabia (Gils et al. 2010).

Throughout the irradiation time with the argon, oxygen glow discharge plasma or mercury lamp, the antibacterial activity of several kinds of bacteria has been examined. Table 2 shows diameter of bacterial inhibition (clear zone) in $\mathrm{mm}$. Bacterial strains number 1, 2, 5, 9 and 10 are Gram-positive bacteria while bacterial strains number $3,4,6,7$ and 8 are Gramnegative bacteria.

\section{Conclusions}

In summary, the irradiation effects of argon, oxygen glow discharge plasma, and mercury lamp on silver and silver/agar nanoparticle samples are studied and compared. The tunability of the SPR position and its characteristics such as FWHM and peak intensity has been investigated. Therefore, the choice of suitable light source leads to controlling the SPR characteristics.

In the present process, glow discharge plasma and mercury lamp irradiation could have high potentials to enhance photochemical reduction method. The irradiation procedure is simple and reproducible and it can be operated at different glow discharge plasma conditions.

The virgin and treated nanoparticles samples exhibited strong antibacterial activity against both the Gram-positive and Gram-negative bacteria. Therefore, the resulting silver and agar/silver nanoparticles samples with antibacterial activity could have high potentials for many applications such as an antibacterial 
Table 2 Diameter of bacterial inhibition zone (clear zone) in $\mathbf{m m}$

\begin{tabular}{|c|c|c|c|c|c|c|c|c|c|c|}
\hline \multicolumn{2}{|c|}{ Sample name } & \multirow{2}{*}{$\begin{array}{l}\text { Untreated } \\
\text { silver NP (A) }\end{array}$} & \multirow{2}{*}{$\begin{array}{l}\text { Untreated } \\
\text { agar/silver } \\
\text { NP (B) }\end{array}$} & \multirow{2}{*}{$\begin{array}{l}\text { Treated }(A) \\
\text { with argon } \\
\text { plasma for } \\
180 \mathrm{~min}\end{array}$} & \multirow{2}{*}{$\begin{array}{c}\text { Treated (B) } \\
\text { with argon } \\
\text { plasma for } \\
180 \text { min }\end{array}$} & \multirow{2}{*}{$\begin{array}{c}\text { Treated (A) } \\
\text { with oxygen } \\
\text { plasma for } \\
150 \text { min }\end{array}$} & \multirow{2}{*}{$\begin{array}{l}\text { Treated (B) } \\
\text { with oxygen } \\
\text { plasma for } \\
150 \text { min }\end{array}$} & \multirow{2}{*}{$\begin{array}{l}\text { Treated (B) } \\
\text { with mercury } \\
\text { lamp at for } \\
40 \mathrm{~min}\end{array}$} & \multirow{2}{*}{$\begin{array}{l}\text { Treated }(A) \\
\text { with mercury } \\
\text { lamp for } \\
15 \mathrm{~min}\end{array}$} & \multirow{2}{*}{$\begin{array}{l}\text { Treated (B) } \\
\text { with mercury } \\
\text { lamp for } \\
15 \mathrm{~min}\end{array}$} \\
\hline No. & Bacteria name & & & & & & & & & \\
\hline 1 & Bacillus cereus & 8 & 8 & 10 & 9 & 8 & 8 & 8 & 8 & 8 \\
\hline 2 & Bacillus subtilis & 9 & 10 & 9 & 9 & 8 & 8 & 8 & 8 & 8 \\
\hline 3 & E. coli & 11 & 14 & 12 & 13 & 11 & 11 & 10 & 11 & 11 \\
\hline 4 & Klebsiella pneumoniae & 15 & 10 & 10 & 10 & 10 & 10 & 10 & 12 & 10 \\
\hline 5 & Micrococcus roseus & 16 & 11 & 10 & 10 & 11 & 10 & 10 & 11 & 10 \\
\hline 6 & Proteus vulgaris & 11 & 8 & 9 & 15 & 9 & 9 & 8 & 8 & 8 \\
\hline 7 & Pseudomonas aeruginosa & 12 & 11 & 14 & 12 & 16 & 14 & 12 & 14 & 12 \\
\hline 8 & Serratia marcescens & 9 & 10 & 9 & 9 & 9 & 9 & 9 & 10 & 9 \\
\hline 9 & Staphylococcus aureus & 17 & 15 & 12 & 11 & 12 & 12 & 11 & 12 & 10 \\
\hline 10 & Streptococcus sp. & 15 & 18 & 13 & 13 & 17 & 14 & 13 & 14 & 12 \\
\hline
\end{tabular}


food packaging and a biomedical application such as wound dressings. However, actual applications of antibacterial nanoparticles require further studies focused on the potential health-hazard of such nanoparticles included products.

\section{Author details}

${ }^{1}$ Physics Department, Faculty of science, Al-Azhar University-Assuit branch, Assuit, Egypt. ${ }^{2}$ Botany and Microbiology Department, Faculty of science,

Al-Azhar University-Assuit branch, Assuit, Egypt.

Received: 26 February 2014 Accepted: 23 July 2014

Published: 19 August 2014

\section{References}

Ahmadi TS, Wang ZL, Green TC, Hengleinet A, El-Sayedal MA (1996) Shape-controlled synthesis of colloidal platinum nanoparticles. Science 272(5270):1924-1925, doi:10.1126/science.272.5270.1924

Amoruso S, Ausanio G, Bruzzese R, Vitiello M, Wang X (2005) Femtosecond laser pulse irradiation of solid targets as a general route to nanoparticle formation in a vacuum. Phys Rev B 71(3):033406-033410, doi:10.1103/PhysRevB.71.033406

Bacławski A, Musielok J (2008) Transition probabilities for some infrared O I spectral lines-Application for determining excitation temperatures in low temperature plasmas. Spectrochim Acta B 63:1315-1319, doi:10.1016/j. sab.2008.10.019

Cobley CM, Skrabalak SE, Campbell DJ, Xia Y (2009) Shape-controlled synthesis of silver nanoparticles for plasmonic and sensing applications. Plasmonics 4(2):171-179, doi:10.1007/s11468-009-9088-0

Duckworth M, Yaphe W (1971) The structure of agar: Part I: Fractionation of a complex mixture of polysaccharides. Carbohydr Res 16(1):189-197, doi:10.1016/S0008-6215(00)86113-3

Freile-Pelegrin Y, Murano E (2005) Agars from three species of Gracilaria (Rhodophyta) from Yucatan Peninsula. Bioresour Technol 96(3):295-302, doi:10.1016/j.biortech.2004.04.010

Gils PS, Ray D, Sahoo PK (2010) Designing of silver nanoparticles in gum arabic based semi-IPN hydrogel. Int J Biol Macromol 46(2):237-244, doi:10.1016/j. ijbiomac.2009.12.014

Guerrero P, Etxabide A, Leceta I, Peñalba M, de la Caba K (2014) Extraction of agar from Gelidium sesquipedale (Rodhopyta) and surface characterization of agar based films. Carbohydr Polym 99:491-498, doi:10.1016/j.carbpol.2013.08.049

Hou W-C, Stuart B, Howes R, Zepp RG (2013) Sunlight-driven reduction of silver ions by natural organic matter: formation and transformation of silver nanoparticles. Environ Sci Tech 47(14):7713-7721, doi:10.1021/es400802w

Kora AJ, Manjusha R, Arunachalam J (2009) Superior bactericidal activity of SDS capped silver nanoparticles: synthesis and characterization. Mater Sci Eng C29(7):2104-2109, doi:10.1016/j.msec.2009.04.010

Kora AJ, Sashidhar RB, Arunachalam J (2010) Gum kondagogu (Cochlospermum gossypium): A template for the green synthesis and stabilization of silver nanoparticles with antibacterial application. Carbohydr Polymer 82(3):670-679, doi:10.1016/j.carbpol.2010.05.034

Labropoulos KC, Niesz DE, Danforth SC, Kevrekidis PG (2002) Dynamic rheology of agar gels: theory and experiments. Part I. Development of a rheological model. Carbohydr Polymer 50(4):393-406, doi:10.1016/S0144-8617(02)00084-X

Link S, El-Sayed MA (2000) Shape and size dependence of radiative, non-radiative and photothermal properties of gold nanocrystals. Int Rev Phys Chem 19 (3):409-453, doi:10.1080/01442350050034180

Mafune F, J-y K, Takeda Y, Tamotsu K (2000) Formation and size control of silver nanoparticles by laser ablation in aqueous solution. J Phys Chem B 104(39):9111-9117, doi:10.1021/jp001336y

McFarland AD, Van Duyne RP (2003) Single silver nanoparticles as real-time optical sensors with zeptomole sensitivity. Nano Lett 3(8):1057-1062, doi:10.1021/jp001336y

Mohan YM, Raju KM, Sambasivudu K, Satyendra S (2007) Preparation of acacia stabilized silver nanoparticles: A green approach. J Appl Polymer Sci 106(5):3375-3381, doi:10.1002/app.26979

NCCLS (1993) National Committee for Clinical Laboratery Standards: Performance standard for antimicrobial Disc susceptibility Tests. Approved Standard NCCLS Publication, Villanova, PA, USA
Pillai ZS, Kamat PV (2004) What factors control the size and shape of silver nanoparticles in the citrate ion reduction method? J Phys Chem B 108(3):945-951, doi:10.1021/jp037018r

Reader J, Sansonetti CJ, Bridges JM (1996) Irradiances of spectral lines in mercury pencil lamps. Appl Optic 35(1):78-83, doi:10.1364/AO.35.000078

Richardson HH, Carlson MT, Tandler PJ, Pedro H, Alexander OG (2009) Experimental and theoretical studies of light-to-heat conversion and collective heating effects in metal nanoparticle solutions. Nano Lett 9(3):1139-1146, doi: $10.1021 / n 18036905$

Shaaban ER, Abdel-Wahab EA, Ahmad M (2013) Optical characterization of As-S thin films induced by plasma immersion $\mathrm{O}$ - ion implantation. Phys Scr 88(1):015703, doi:10.1088/0031-8949/88/01/015703

Shalaev VM (2002) Optical properties of nanostructured random media, vol 82. Topics in Applied Physics, Springer Verlag, Berlin, doi:10.1007/3-540-44948-5

Zhen SJ, Zhang ZY, Li N, Zhang ZD, Wang J, Li CM, Zhan L, Zhuang HL, Huang CZ (2013) UV light-induced self-assembly of gold nanocrystals into chains and networks in a solution of silver nitrate. Nanotechnology 24(5):055601, doi:10.1088/0957-4484/24/5/055601

doi:10.1186/2193-1801-3-443

Cite this article as: Ahmad et al:: Irradiation of silver and agar/silver nanoparticles with argon, oxygen glow discharge plasma, and mercury lamp. SpringerPlus 2014 3:443.

\section{Submit your manuscript to a SpringerOpen ${ }^{\odot}$ journal and benefit from:}

- Convenient online submission

Rigorous peer review

- Immediate publication on acceptance

- Open access: articles freely available online

- High visibility within the field

- Retaining the copyright to your article

Submit your next manuscript at $>$ springeropen.com 